\title{
Urolithin a attenuates IL-1 $\beta$-induced inflammatory responses and cartilage degradation via inhibiting the MAPK/NF-KB signaling pathways in rat articular chondrocytes
}

Sheng-long Ding ${ }^{1+} \mathbb{D}$, Zhi-ying Pang ${ }^{2+}$, Xue-mei Chen $^{3+}$, Zheng Li ${ }^{2}$, Xin-xin Liu ${ }^{2}$, Qi-lin Zhai', Jun-ming Huang ${ }^{2^{*}}$ and Zhi-yong Ruan ${ }^{1^{*}}$

\begin{abstract}
Background: Osteoarthritis $(\mathrm{OA})$ is characterized by inflammation and extracellular matrix (ECM) degradation and is one of the most common chronic degenerative joint diseases that causes pain and disability in adults. Urolithin A (UA) has been widely reported for its anti-inflammatory properties in several chronic diseases. However, the effects of UA on OA remain unclear. The aim of the current study was to investigate the anti-inflammatory effects and mechanism of UA in interleukin-1 $\beta$ (IL-1 $\beta$ )-induced chondrocytes.

Results: No marked UA cytotoxicity was noted, and UA protected cartilage from damage following IL-1 $\beta$ stimulation in micromasses. Moreover, UA promoted the expression of anabolic factors including Sox-9, Collagen II, and Aggrecan while inhibiting the expression of catabolic factors such as matrix metalloproteinases (MMPs) and a disintegrin and metalloproteinase with thrombospondin motifs 4 (ADAMTS-4) in rat chondrocytes. Protective effects of UA were also observed in ex vivo organ culture of articular cartilage. Mechanistically, IL-1 $\beta$ significantly activated and upregulated the expression of p-ERK 1/2, p-JNK, p-P38, and p-P65, while UA protected chondrocytes against IL-1 $\beta$-induced injury by activating the mitogen-activated kinase (MAPK)/nuclear factor-KB (NF-KB) signaling pathways.

Conclusion: Our results provide the evidence that UA could attenuate IL-1 $\beta$-induced cell injury in chondrocytes via its anti-inflammatory action. $\cup A$ may be a promising therapeutic agent in the treatment of $O A$.
\end{abstract}

Keywords: Urolithin a, Osteoarthritis, NF-KB, MAPK, Inflammation

\footnotetext{
*Correspondence: mhyper@sina.com; xyrzy818@163.com

${ }^{\dagger}$ Sheng-long Ding, Zhi-ying Pang and Xue-mei Chen contributed equally to this work.

${ }^{2}$ Department of Orthopaedic Surgery, Zhongshan Hospital, Fudan University, 180 Feng Lin Road, Xuhui District, Shanghai 200032, China

'Department of Orthopaedic Surgery, Qingpu Branch of Zhongshan Hospital,

Fudan University, 1158 Gong Yuan Dong Road, Qingpu District, Shanghai

201700, China

Full list of author information is available at the end of the article
}

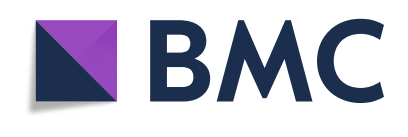

( ) The Author(s). 2020 Open Access This article is licensed under a Creative Commons Attribution 4.0 International License, which permits use, sharing, adaptation, distribution and reproduction in any medium or format, as long as you give appropriate credit to the original author(s) and the source, provide a link to the Creative Commons licence, and indicate if changes were made. The images or other third party material in this article are included in the article's Creative Commons licence, unless indicated otherwise in a credit line to the material. If material is not included in the article's Creative Commons licence and your intended use is not permitted by statutory regulation or exceeds the permitted use, you will need to obtain permission directly from the copyright holder. To view a copy of this licence, visit http://creativecommons.org/licenses/by/4.0/. The Creative Commons Public Domain Dedication waiver (http://creativecommons.org/publicdomain/zero/1.0/) applies to the data made available in this article, unless otherwise stated in a credit line to the data. 


\section{Introduction}

Osteoarthritis (OA) is one of the most common forms of chronic degenerative joint disease and affects tens of millions of people around the world [1]. The main characteristic features observed in $\mathrm{OA}$ include progressive loss and destruction of articular cartilage, thickening of the subchondral bone, osteophyte formation, and synovial inflammation Multiple factors contribute to the initiation and progression of OA, such as aging, heredity, obesity, abnormal metabolism, joint injury, osteoporosis, and joint malformation [2, 3].

At the cellular and molecular levels, inflammation and inflammatory mediators play crucial roles in initiating and accelerating OA development $[4,5]$. A growing body of evidence suggests that interleukin-1 $\beta$ (IL-1 $\beta$ ), tumor necrosis factor-alpha (TNF- $\alpha$ ), and IL- 6 are found in OA cartilage [6]. Among these inflammatory cytokines, the effect of IL-1 $\beta$ was widely explored because of its vital role in inflammatory responses. The pro-inflammatory cytokine IL- $1 \beta$ is a master regulator of inflammation that has been reported to directly participate in the generation of multiple inflammatory mediators [3]. When chondrocytes are stimulated by IL-1 $\beta$, they produce metalloproteinases (MMPs), a metalloproteinase with a thrombospondin type 1 motifs (ADAMTS), and some inflammation-associated proteins including inducible nitric oxide synthase (iNOS) and cyclooxygenase-2 (COX-2), which trigger the alteration of cartilage from the normal homeostatic state toward a catabolic state and eventually leads to extracellular matrix (ECM) degradation $[7,8]$. Therefore, targeting IL-1 $\beta$-induced catabolic metabolism and inflammatory responses may be an effective strategy to delay OA progression.

Urolithin A (UA) is metabolized by intestinal microbiota from Ellagitannins (ETs) and Ellagic acid (EA) in the gut $[9,10]$. According to previous studies, ETs and EA may inhibit the inflammatory response. Dietary consumption of EA-rich food has been demonstrated to suppress inflammatory cytokine release in the brains of Alzheimer's disease mice [11]. Similarly, EA protects against cisplatin-induced kidney nephrotoxicity by inhibiting renal inflammation and apoptosis [12]. Nevertheless, EA is poorly absorbed and quickly eliminated, and the biological activity of EA is controversial. Interestingly, recent published studies have described the biological effects of UA, including anti-proliferation in cancer, antiinflammation, anti-oxidant activity, improved lipid metabolism [13-15]. UA inhibits the catabolic effect of TNF- $\alpha$ on nucleus pulposus cells and alleviates intervertebral disc degeneration in vivo [16]. Moreover, UA can protect skeletal muscle against acute inflammation in vitro and in vivo [17]. Mechanistically, UA could significantly inhibit the activation of NF- $\mathrm{kB}$ induced by IL-1 $\beta$ in colon fibroblasts [18]. Meanwhile, $\mathrm{Fu}$ et al. investigated the anti- inflammatory effect of UA in human OA and revealed the underlying mechanism by blockage of PI3K/Akt/NF-kB pathway [19]. Although the potential anti-inflammatory role of UA has been extensively investigated, there is limited knowledge whether UA has other potential therapeutic targets to attenuate the pathogenesis of OA. In this study, we investigated the anti-inflammatory role of UA by attenuating IL- $1 \beta$-induced degradation of Collagen II and Aggrecan and by reducing the production of inflammatory mediators via the ERK, JNK, P38, and NF- $\mathrm{B}$ p pathways in rat chondrocytes.

\section{Materials and methods \\ Antibodies and reagents}

Urolithin A (CAS No. 1143-70-0) was bought from Cayman Chemical (Ann Arbor, MI, USA). Recombinant rat IL-1 $\beta$ (400-01B) was purchased from (Peprotech, Suzhou, China). Fetal bovine serum (FBS) was provided by Gibco Life Technologies (Grand Island, NY, USA). Antibodies against antiphospho-ERK (\#4370), ERK (\#9102), antiphospho-JNK (\#4668), JNK (\#9252), antiphospho-p38 (\#4511), p38 (\#8690), antiphosphop65 (\#3033), p65 (\#8242), COX2 (\#12282), iNOS (\#13120) Sox-9 (\#82630) were bought from Cell Signaling Technology (Danvers, MA, USA). Antibodies against MMP3 (ab52915), MMP13 (ab39012), Collagen II (ab34712), aggrecan (ab36861) were purchased from Abcam (Cambridge, UK). Horseradish peroxidase (HRP)conjugated glyceraldehyde 3-phosphate dehydrogenase (GAPDH) (ET1702-66) and HRP-linked goat anti-rabbit (HA1001) were supplied by Huabio (Hangzhou, China). Phalloidin was provided by Beyotime (Shanghai, China). Cy3- conjugated goat anti-rabbit secondary antibody (BA1032) and 4',6-diamidino-2-phenylindole (DAPI) (AR1177) were purchased from Boster (Wuhan, China). Other reagents were of the highest commercial grade and were purchased from Sigma Chemical (St. Louis, MO, USA).

\section{Cell culture}

Primary chondrocytes were obtained from knee joints cartilage of 2-week-old Sprague Dawley rats. The detailed procedure was performed according to a previously described method [20]. Briefly, cartilage of the knee joint was isolated and cut into pieces and then incubated in $0.5 \%$ trypsin-EDTA (containing $0.5 \mathrm{~g} / \mathrm{L}$ of trypsin (1:250) and $0.2 \mathrm{~g} / \mathrm{L}$ EDTA.4 $\mathrm{Na}$ in $0.85 \%$ saline solution) for $30 \mathrm{~min}$ and subsequently $0.2 \%$ collagenase for $24 \mathrm{~h}$ at $37^{\circ} \mathrm{C}$. The chondrocytes were collected and cultured in Dulbecco's minimum essential medium: F12 medium containing 10\% FBS with humid air with 5\% $\mathrm{CO}_{2}$ at $37{ }^{\circ} \mathrm{C}$. Cells were trypsinized with $0.5 \%$ trypsinEDTA and passaged at a ratio of 1:3 when cell density reaches $75 \%$, and the medium was changed every 2 days. 
Chondrocytes at passage 3 were utilized in the subsequent experiments.

\section{Cell viability assay}

The Cell Counting Kit-8 (CCK8, Dojindo, Japan) was utilized to analyze cell viability. Firstly, chondrocytes were seeded in 96-well plates at a density of $1 \times 10^{4}$ cells/well. After $24 \mathrm{~h}$ of adhesion, cells were then treated with IL-1 $\beta$ alone or with UA at different concentrations. Cell viability was carried out after cultivating for 1,3 , and 7 days. In brief, $10 \mu \mathrm{l}$ CCK- 8 solution dissolved in $100 \mu \mathrm{l}$ culture medium was added into each well and then incubated in the dark at $37^{\circ} \mathrm{C}$ for $1.5 \mathrm{~h}$. The absorbance of the solution was recorded at $450 \mathrm{~nm}$ using a plate reader (BioTek, Winooski, VT, USA).

\section{Micromass culture}

All procedures were performed as previously described $[21,22]$. Briefly, the chondrocytes were suspended in medium with $10 \% \mathrm{FBS}, 0.25 \%$ penicillin-streptomycin, and $0.25 \%$ L-glutamine, and plated at a density of $2.5 \times$ $10^{5}$ cells $/ 10 \mu \mathrm{l}$ in 24 -well plates. Four hours later, the medium was added into the plate with IL-1 $\beta$ alone or with IL-1 $\beta$ with UA for 2 days. Then the micromasses were stained with Alcian Blue.

\section{Western blotting analysis}

Chondrocytes were cultured in a sterile six-well plates at $37{ }^{\circ} \mathrm{C}$ with $5 \% \mathrm{CO}_{2}$. After reaching $80 \%$ density, the cells were exposed to IL-1 $\beta$ alone or with UA. The total proteins were obtained from stimulated or control chondrocytes using radioimmunoprecipitation assay lysis buffer containing $1 \%$ proteinase inhibitor and $1 \%$ phosphatase inhibitor cocktail for $30 \mathrm{~min}$ on ice at the indicated time points. Protein concentrations were measured using BCA protein assay kits (Boster). Then, $40 \mu \mathrm{g}$ of protein was separated on $12 \%$ sodium dodecyl sulfatepolyacrylamide gels and transferred to polyvinylidene fluoride membranes (Millipore, Burlington, MA, USA), blocked with 5\% bovine serum albumin (BSA) in Trisbuffered saline with $0.1 \%$ Tween-20 (TBS-T) and incubated with primary antibody (2\% BSA in TBS-T) overnight at $4{ }^{\circ} \mathrm{C}$. Subsequently, the membrane was washed with TBS-T and incubated with the corresponding secondary antibodies for $2 \mathrm{~h}$ at room temperature. Finally, the protein bands were visualized with western ECL Substrate Kits (Yseasen, Shanghai, China) on a Tanon imaging system, and grayscale images were analyzed with Image (National Institutes of Health, Bethesda, MD, USA)/Olympus (Tokyo, Japan) software.

Total RNA extraction and quantitative real-time RT-PCR Total RNA was extracted by a total RNA extraction kit (Omega Bio-tek, Norcross, GA, USA) from chondrocytes exposed to IL-1 $\beta$ alone or with UA in accordance with the manufacturer's instructions. RNA purity and concentration were determined by a spectrophotometer (Thermo Fisher Scientific, Waltham, MA, USA). Complementary DNA (cDNA) was synthesized from total RNA and amplified with SYBR Green Master Mix in an ABI PRISM 7500 PCR Sequence Detection System (Applied Biosystems, Foster City, CA, USA) according to following condition: $30 \mathrm{~s}$ of denaturation followed by 40 cycles of $94{ }^{\circ} \mathrm{C}$ for $5 \mathrm{~s}$ and $60^{\circ} \mathrm{C}$ for $35 \mathrm{~s}$. The melting curve was generated to test for primer dimer formation and false priming for each reaction. Relative expressions of gene-specific products were analyzed using the comparative $\mathrm{Ct}\left(2^{-\Delta \Delta \mathrm{Ct}}\right)$ method and normalized to the reference gene GAPDH. The sequences of primers constructed were as follows: ADAMTS4: forward (CCGTTCCGCTCCTG TAACACTAAG), reverse (AGGTCGGTTCGGTGGTTG TAGG); MMP9: forward (CTACACGGAGCATGGCAA CGG), reverse (TGGTGCAGGCAGAGTAGGAGTG); Col2a1: forward (ACGCTCAAGTCGCTGAACAACC), reverse (ATCCAGTAGTCTCCGCTCTTCCAC); GAPDH: forward (GACAATTTTGGCATCGTGGA), reverse (ATGCAGGGATGATGTTCTGG).

\section{Immunofluorescence}

Chondrocytes were plated in 12-well plates. When the density reached $80 \%$, the cells were stimulated with IL- $1 \beta$ alone or with UA. Next, cells were fixed in $4 \%$ paraformaldehyde for $15 \mathrm{~min}$ at room temperature. Subsequently, the cells were permeabilized in phosphate-buffered saline (PBS) containing $0.3 \%$ Triton $\mathrm{X}-100$ for $15 \mathrm{~min}$ and then blocked with 5\% BSA for $30 \mathrm{~min}$. Cells were then incubated with anti-P65 (1:200 dilution) in a humid chamber overnight at $4{ }^{\circ} \mathrm{C}$. The next day, the plates were washed three times with PBS and then incubated with Cy3conjugated goat antirabbit secondary antibody (1:100 dilution) at $37^{\circ} \mathrm{C}$ for $1 \mathrm{~h}$ in the dark. Finally, cells were stained with phalloidin and DAPI. Images were acquired using an inverted fluorescence microscope (Olympus) with identical acquisition settings, and the results were statistically analyzed using ImageJ software.

\section{Ex vivo organ culture of rat articular cartilage}

All experimental protocols were approved by the Committee of Ethics of Animal Experiments at Zhongshan Hospital, Fudan University, China. Cartilage explants were obtained from the knee joints of 4-week-old Sprague Dawley rats that were group-housed at $20 \pm$ $5{ }^{\circ} \mathrm{C}$ ( $55 \pm 5 \%$ humidity) on a 12 -h light/dark cycle with free access to standard chow and water. The detailed procedure was described in a published protocol [23]. Initially, the explants were cultured in medium containing $10 \% \mathrm{FBS}$ at $37^{\circ} \mathrm{C}$ with $5 \% \mathrm{CO}_{2}$ for 2 days. Then, the explants were cultured in medium (10\% FBS and $0.25 \%$ 
penicillin-streptomycin) containing IL-1 $\beta$ and/or IL-1 $\beta$ with UA for 3 additional days. Next, explants were collected and fixed in $4 \%$ paraformaldehyde, sectioned at $6 \mu \mathrm{m}$, and stained with hematoxylin and eosin (H\&E), Safranine O-Fast Green (S-O Fast green), or Alcian Blue, then we used the Osteoarthritis Research Society International (OARSI) scoring system with double blindness as described previously to evaluate the destruction of articular cartilage, scoring including the matrix staining, cartilage tissue structure, chondrocyte clusters, and surface integrity $[24,25]$. Further, Collagen type II and aggrecan were analyzed by immunochemistry and The percentages of Collagen II, Aggrecan positive cells in each section were quantified by Image Pro Plus. All stained sections were imaged using an upright microscope (Olympus).

\section{Statistical analysis}

The experiments were performed at least three times. All data are presented as mean \pm standard deviation (SD). Statistical analyses were performed using GraphPad Prism software (GraphPad Inc., San Diego, CA, USA) and SPSS 18.0 (IBM, Armonk, NY, USA). For differences among treatments, Student's $t$-tests were used for the comparisons between two groups, and data involving more than two groups were analyzed by oneway analysis of variance followed by Tukey post hoc tests. $P$ values less than 0.05 were considered statistically significant.

\section{Results}

Cell viability after IL-1 $\beta$ or/and UA treatment

First, we examined the potential toxicity of UA on chondrocytes with CCK8 assays. As shown in Fig. 1a, UA had no significant effect on chondrocyte viability and proliferation at concentrations of $1,5,7.5$, or $15 \mu \mathrm{M}$ for 1,3 , or 7 days. However, chondrocyte activity decreased by $\sim 50 \%$ compared with the control group $(P<0.05)$ when the concentration reached $30 \mu \mathrm{M}$, indicating that a high concentration of UA may inhibit cell activity. Therefore, we set the maximum concentration of UA to $15 \mu \mathrm{M}(1,7.5$, $15 \mu \mathrm{M})$ for subsequent experiments. When chondrocytes were treated with IL-1 $\beta$ for 1,3 , or 7 days, as shown in Fig. $1 \mathrm{~b}$ and $\mathrm{c}$, there were no significant changes in cell viability with increasing concentrations of IL-1 $\beta(<30 \mathrm{ng} / \mathrm{ml})$ with and without UA $(<15 \mu \mathrm{M})$. To investigate whether UA protects against cell damage induced by IL- $1 \beta$, cartilage micromasses were co-incubated with $20 \mathrm{ng} / \mathrm{ml} \mathrm{IL-1 \beta}$ and various concentrations of UA from 1 to $15 \mu \mathrm{M}$ for 2 days and then stained with Alcian Blue. UA markedly ameliorated IL-1 $\beta$-induced degradation of cartilage matrix in a dose-dependent manner (Fig. 1d). These results suggest that no marked UA cytotoxicity occurred in chondrocytes, and UA partially protected against IL- $1 \beta$-induced cartilage matrix degradation.

\section{UA inhibited IL-1 $\beta$-induced ECM catabolism in chondrocytes}

ECM gene expression levels were detected with RT-qPCR after treatment with various UA concentrations $(0,1,7.5$,

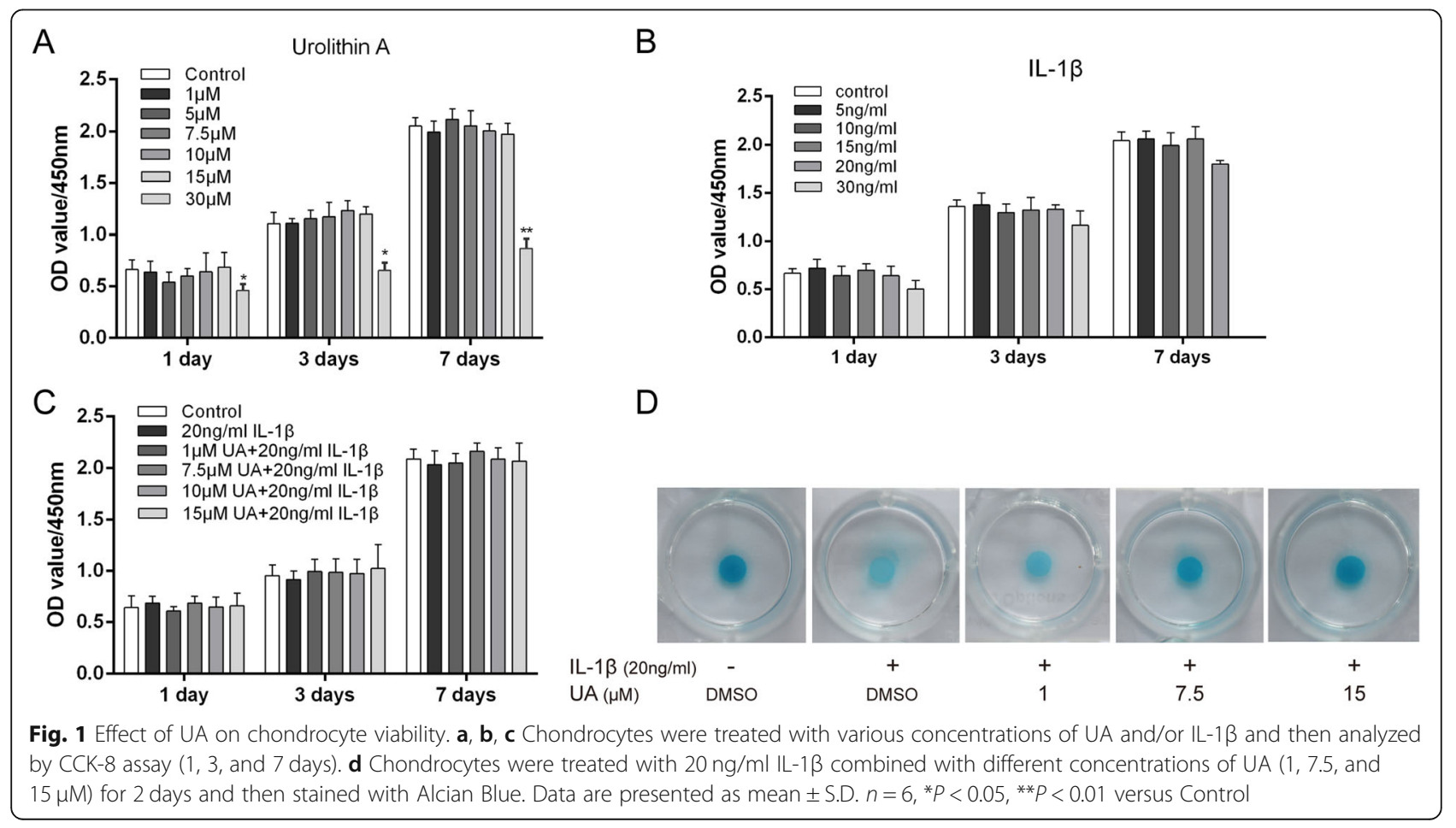


$10 \mu \mathrm{M})$ for $48 \mathrm{~h}$. MMP9 and ADAMTS4 are matrixdegrading enzymes, and Collagen II could antagonize this effect and promote ECM anabolism. MMPs (MMP3 and MMP13) are catabolic enzymes of Collagen II and Aggrecan. As shown in Fig. 2g and h, MMP9 and ADAMTS4 mRNA expression markedly increased in a dose-dependent manner. UA obviously suppressed the overproduction of MMP9 and ADAMTS4 mRNA induced by IL-1 $\beta$ stimulation. Meanwhile, UA reversed the downregulated gene expression of Collagen II in the IL-1 $\beta$ stimulated condition. However, UA did not affect the expression of these genes at the lowest concentration $(1 \mu \mathrm{M})$. The effect of UA on IL-1 $\beta$-induced MMP 3 and MMP13 production were measured by western blot. UA treatment partially reduced protein expression of MMP 3 and MMP13 compared to cells treated with IL-1 $\beta$ alone (Fig. 2a).

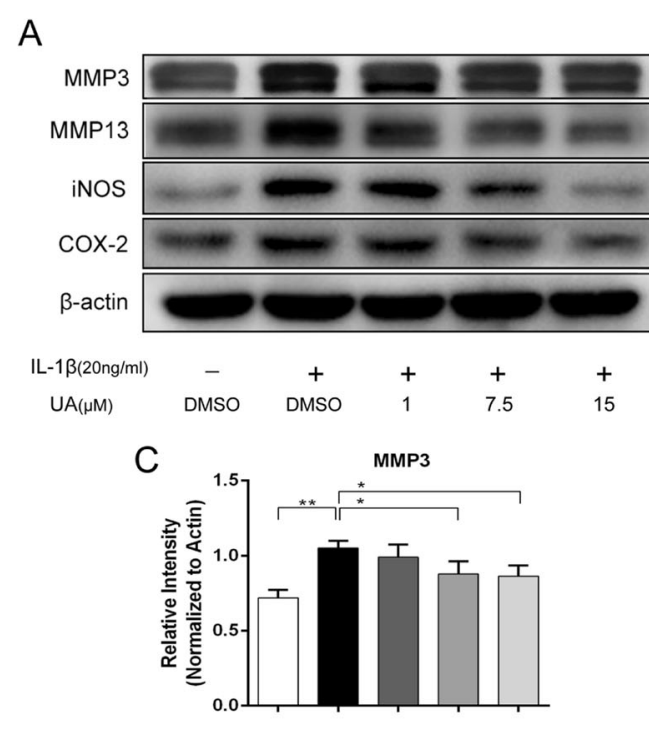

$\mathrm{E}$

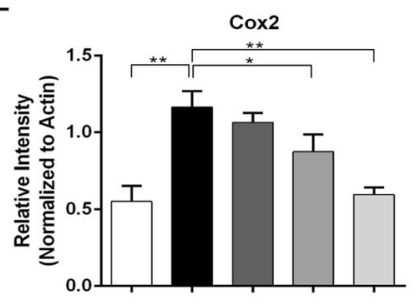

G

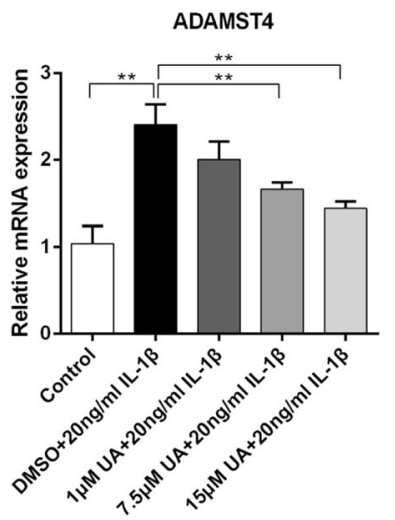

B

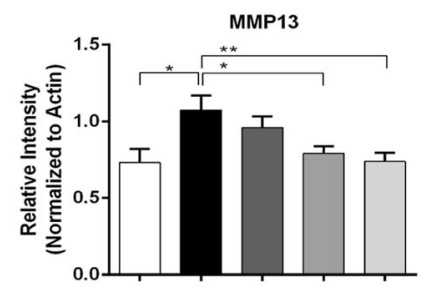

D

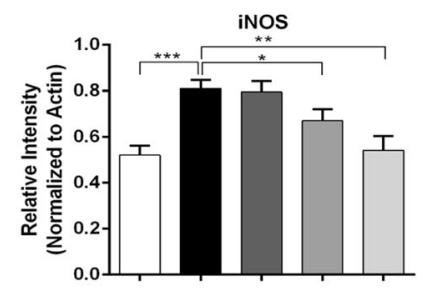

$\mathrm{F}$

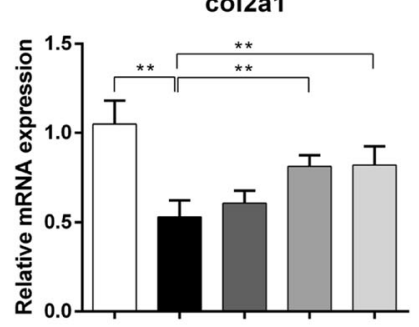

$\mathrm{H}$

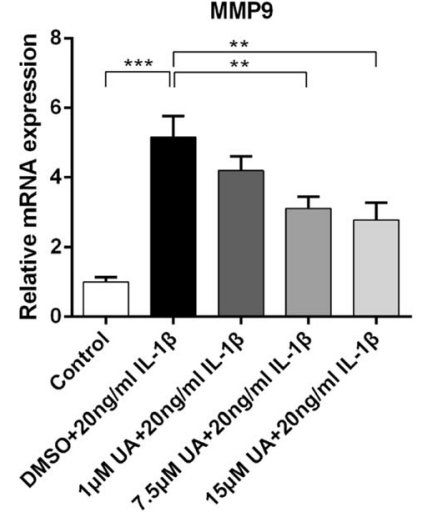

Fig. 2 Effect of UA on IL-1 $\beta$ induced expression of iNOS, COX2, and MMPs. Chondrocytes were treated with IL-1 $\beta$ (20 ng/ml) alone or UA (1, 7.5 and $15 \mu \mathrm{M})$ in combination with $\mathrm{LL}-1 \beta(20 \mathrm{ng} / \mathrm{ml})$ for $48 \mathrm{~h}$. a Protein expression of $\mathrm{NOS}$, COX2, MMP3, and MMP13 were determined by western blot. b, c, d, e Relative protein expression of iNOS, COX2, MMP3, and MMP13 shown as histograms. $\mathbf{f}, \mathbf{g}$, $\mathbf{h}$ Relative gene expression of MMP9, ADAMTS4, and Collagen 2a1 were measured by RT-qPCR. Data are presented as mean \pm S.D. $n=6 .{ }^{*} P<0.05,{ }^{* *} P<0.01,{ }^{* * *} P<0.001$ versus the IL-1ß group 
UA prevented IL-1 $\beta$-induced degradation of Sox-9, collagen II, and Aggrecan

To evaluate chondrocyte degeneration, we investigated ECM replacement by chondrocytes under IL- $1 \beta$ stimulation with or without UA pretreatment by western blot analysis. Collagen II and Aggrecan are the two main components of cartilage matrix responsible for the anti-compression and shock absorption capabilities of cartilage under mechanical loading. Fig. 3a-c show that IL-1 $\beta$ significantly decreased protein expression of Collagen II $(P<0.05)$ and Aggrecan $(P<0.01)$. However, these alterations were reversed by pretreatment with UA, especially at the highest concentration of $15 \mu \mathrm{M}$. The key regulator of Collagen II synthesis is Sox-9, and UA could prevent its degradation induced by IL-1 $\beta(P<0.01$, Fig. $3 \mathrm{a}$ and $\mathrm{d})$. This result was consistent with the RT-qPCR findings.

\section{UA suppressed IL-1 $\beta$-induced expression of iNOS and COX2 in chondrocytes}

Protein expression levels of iNOS and COX-2 were quantified to examine the extent of IL-1 $\beta$-induced inflammation and evaluate whether it is attenuated by UA. Chondrocytes were pretreated with different concentrations of UA $(1-15 \mu \mathrm{M})$ for $2 \mathrm{~h}$ and then simulated with or without IL-1 $\beta(20 \mathrm{ng} / \mathrm{ml})$ for $48 \mathrm{~h}$. Western blotting was performed to detect protein expression of the inflammatory mediators iNOS and COX2. As shown in Fig. 2a, IL-1 $\beta$ stimulation significantly increased iNOS and COX2 production. However, UA inhibited the excessive production of these mediators. Our results demonstrate that UA co-treatment significantly $(P<0.05)$ and dose dependently decreased the inflammation induced by IL- $1 \beta$. However, the lowest dose of UA $(1 \mu \mathrm{M})$ had no protective effects $(P>0.05)$.

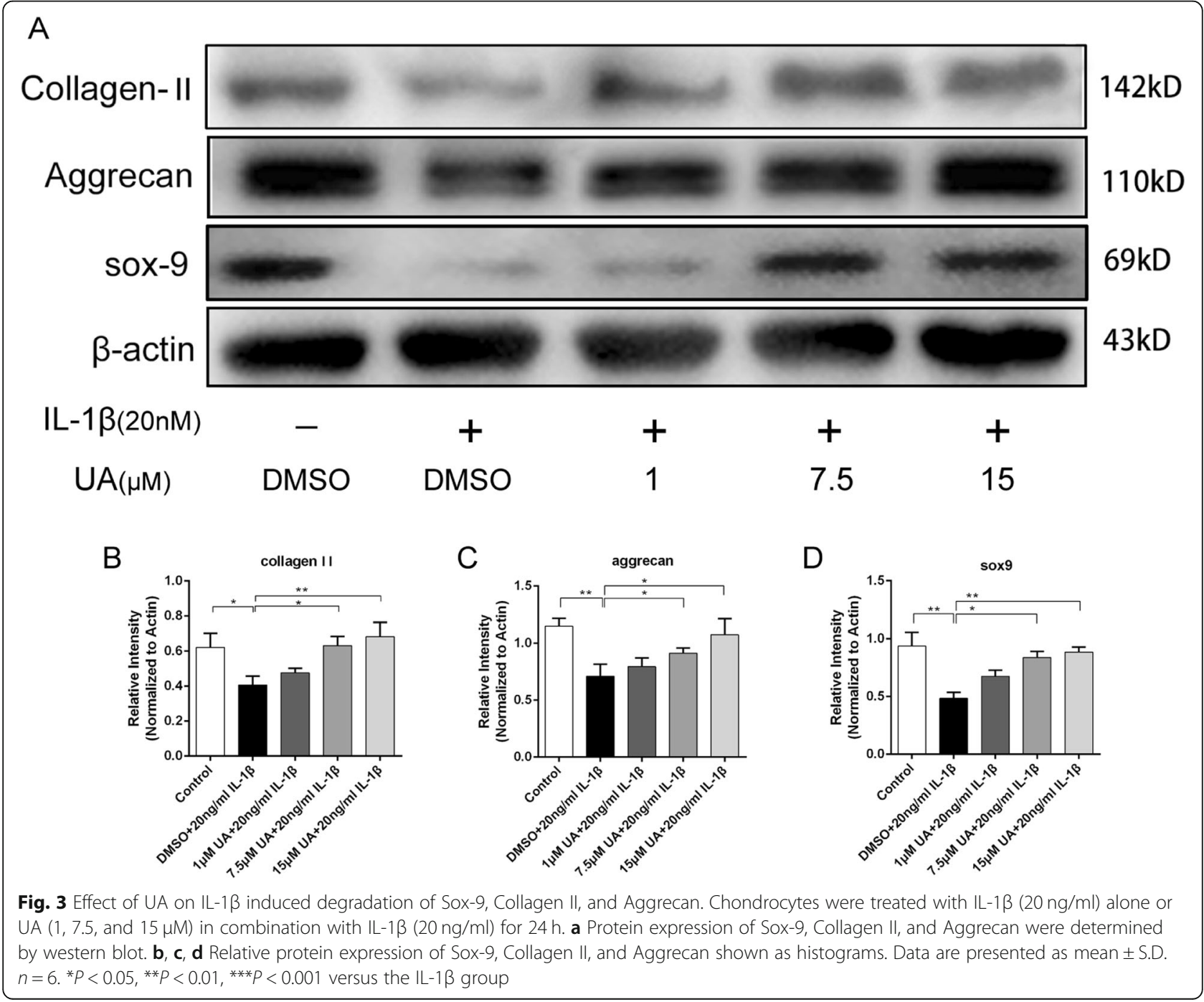




\section{Effect of UA on IL-1 $\beta$-induced activation of the MAPK pathway}

Previous studies have demonstrated that IL- $1 \beta$ could trigger inflammation by activating the mitogen-activated protein kinase (MAPK) pathway [26]. Specifically, MAPK signaling mediates inflammation responses and cartilage degradation in the pathogenesis of OA. To clarify the mechanism of action underlying UA protection, MAPK activity evaluated using western blot analysis. The phosphorylation levels of ERK, JNK, and p38 were significantly upregulated compared to the control group after treatment with IL-1 $\beta$ for $2 \mathrm{~h}(P<0.01)$. Notably, UA could suppress the upregulated phosphorylation of ERK1/2, JNK, and p38 in a concentration-dependent manner (Fig. 4a-d). These results suggest that UA protects chondrocytes against IL- $\beta$-induced inflammation injury by inhibiting the phosphorylation of MAPK pathway members.

\section{UA inhibited IL-1 $\beta$-mediated activation of the NF-KB pathway}

To further explore the anti-inflammatory mechanism of UA, immunofluorescence and western blot analyses of NF- $\kappa B$ p65 were performed to evaluate the effect of UA on the NF- $\mathrm{KB}$ pathway. IL- $1 \beta$ significantly up-regulated p65 phosphorylation $(P<0.01)$. As expected, UA remarkably inhibited IL-1 $\beta$-induced NF- $\mathrm{kB}$ activation in a dose-dependent manner (Fig. 4a and b). However, it is worth noting that phosphorylated p65 was lower than in the control group and the inhibitory effect of UA did not increase at concentrations $>7.5 \mu \mathrm{M}$. Immunofluorescence showed that most p65 was present in the cytoplasm in control cells. However, as shown in Fig. 5a and b, IL-1 $\beta$ treatment significantly increased p65 fluorescence intensity, indicating that NF- $\mathrm{KB}$ activation induced its nuclear translocation and subsequent transcription of inflammatory mediators. Moreover, chondrocytes treated with $20 \mathrm{ng} / \mathrm{ml} \mathrm{IL-1 \beta}$ for $2 \mathrm{~h}$ exhibited nearly $80 \%$ activated p65 was activated, as demonstrated by an $\sim 8$ fold increase in fluorescence intensity compared to the control group. However, UA pretreatment inhibited p65 translocation into the nucleus (Fig. 5a and b). This observation was consistent with the western blot results. Collectively, these findings suggest that UA protects chondrocytes against IL- $\beta$-induced inflammation injury by inhibiting phosphorylation of a member of the NF- $\mathrm{kB}$ pathway (p65).

\section{UA inhibited damage in cartilage explant culture}

We used an ex vivo culture model of cartilage explants from nine 4-week-old rats to evaluate effect of UA on cartilage degradation. The cartilage explants were treated with UA $(15 \mu \mathrm{M})$ with or without IL-1 $\beta$ stimulation (30 $\mathrm{ng} / \mathrm{ml}$ ) for 3 days. The explants were divided into three groups: (1) control, (2) IL-1 $\beta$ stimulated $(30 \mathrm{ng} / \mathrm{ml})$, and
(3) IL-1 $\beta$ plus $(30 \mathrm{ng} / \mathrm{ml})$ UA $(15 \mu \mathrm{M})$. Histopathological changes in cartilage were evaluated by $\mathrm{H} \& \mathrm{E}, \mathrm{S}-\mathrm{O}$ Fast green, and Alcian blue staining. As shown in Fig. 6a, h \&E and S-O Fast Green staining revealed normal structure of cartilage including smooth and intact surfaces and normal morphology and numbers of well-organized chondrocytes in the control group. However, the IL- $1 \beta$ treated group had apparent morphological changes including rough surfaces (black arrow), clustered and disorganized chondrocytes (black triangle), obvious hypocellularity, and loss of Safranin-O staining compared with the control group. Notably, OARSI scores of the cartilage showed cartilage damage was significantly attenuated by treatment with UA (Fig. 6b). Alcian Blue staining for glycosaminoglycan (GAG) distribution. The control group showed the strongest positive expression of GAG, indicating a sound chondroprotective effect. However, the IL-1 $\beta$ group showed the loss of GAG from the superficial zone to the deep zone of articular cartilage (Fig. 6c). Intriguingly, these changes were slightly decreased by UA. Immunohistochemistry showed that the expression of Collagen II and Aggrecan were reduced by IL-1 $\beta$ (Fig. 6d). Apparently, UA administration could effectively reverse the pathological changes with significantly (Fig. 6, f), which was consistent with the western blot results. Taken together, these results indicate that both the structure and ECM of cartilage tissues were better preserved in the UA-treated group.

\section{Discussion}

OA is traditionally considered a mechanically induced chronic condition and affects more than $25 \%$ of the population over 18 years old. Although the occurrence of $\mathrm{OA}$ is closely related to multiple factors, the exact pathogenesis remains unclear [27]. Additionally, multiple non-surgical regimens used for OA were limited for many aspects including recurrent side effects and variable rates of success. Thus, a safe and effective drug with a certain molecular target is in urgent need to alleviate cartilage degradation. The gut microbiota and its metabolites could affect multiple organs and contribute to disease progression $[28,29]$. Short-chain fatty acids, the main products of intestinal bacterial fermenting dietary fiber, intrigued researchers because of its potential role in the prevention and treatment of metabolic syndrome, bowel disorders, and cancer [30,31]. UA is a metabolite derived from EA and ETs with a lower molecular weight and better bioavailability compared to its precursors; it is thought to play a protective role in chronic disease with broad spectrum of anti-inflammatory effects. Here, we demonstrated that UA prevented IL- $1 \beta$-induced damage of ex vivo cartilage explants. Under IL- $1 \beta$ stimulation, UA also attenuated the increased expression of cartilage catabolic enzymes (iNOS, COX2, MMPs) and 


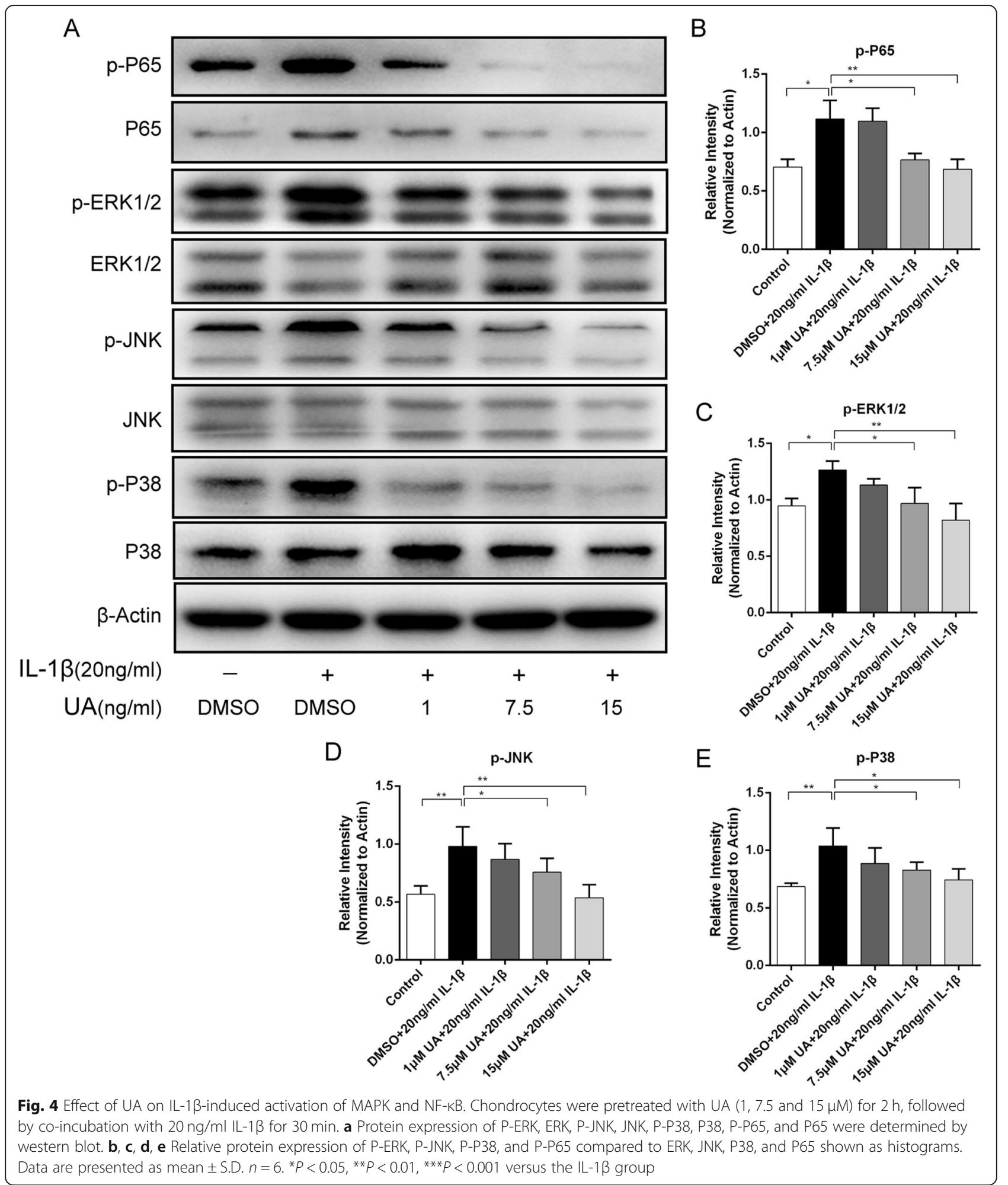

restored the decreased expression of Sox-9 in rat chondrocytes. Moreover, IL-1 $\beta$-induced degradation of Collagen II and aggrecan was attenuated by UA. Finally, we found that the MAPK and NF-kB pathways were involved in the protective effects of UA. Collectively, our results suggested that UA may be a promising therapeutic strategy for OA.

Numerous studies have implicated the pro-inflammatory cytokine IL-1 $\beta$ as a vital factor in OA, because it is significantly increased in the synovial fluid of OA patients. Anti- 


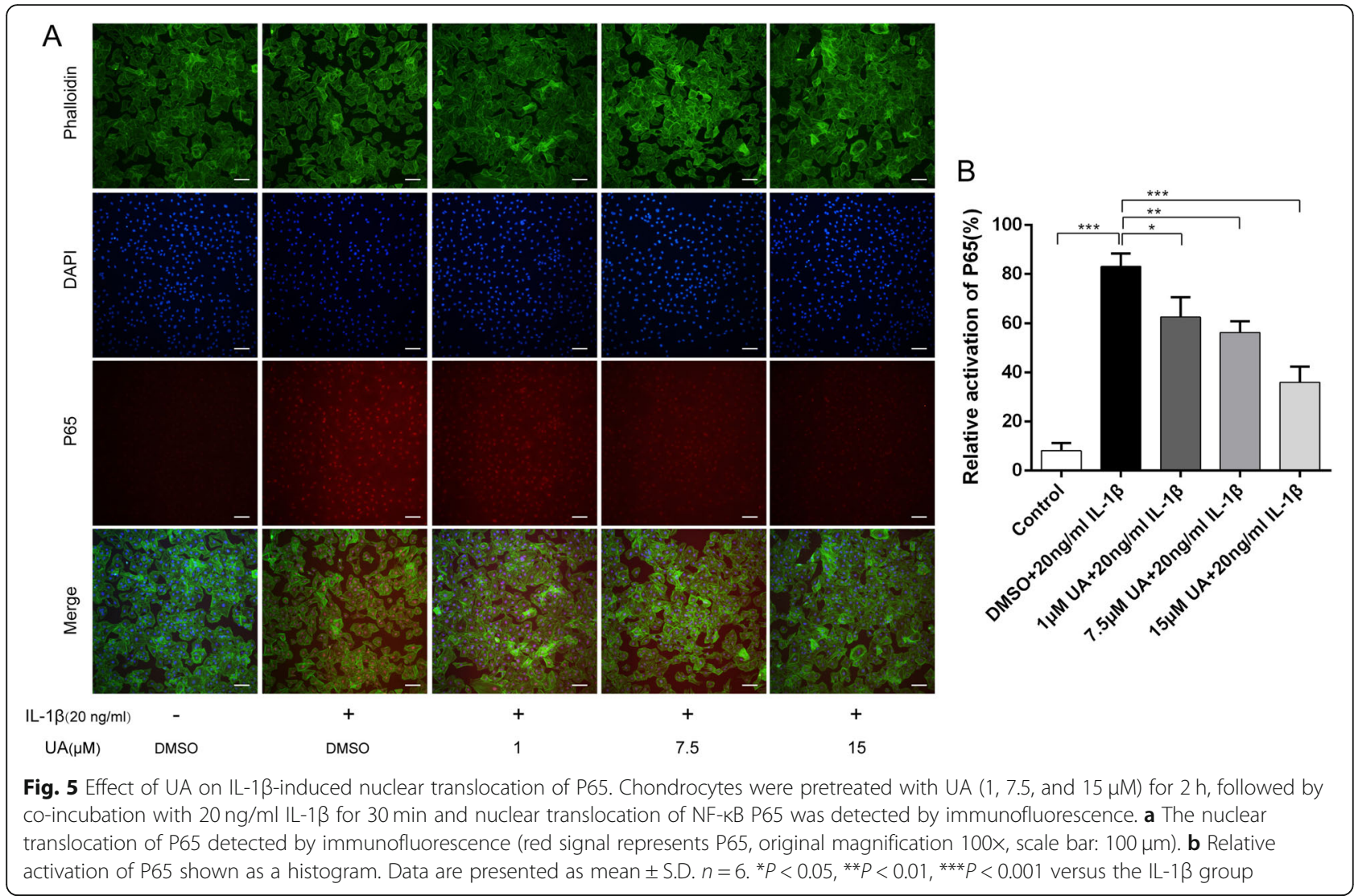

inflammatory treatment plays a key role in alleviating OA symptoms. A recent study demonstrated that the Liraglutide (GLP-1 agonist) ameliorates cartilage degeneration in a rat model of knee osteoarthritis with anti- inflammatory activity [32]. Valproic acid and butyrate were widely reported as latent therapeutic agents for OA due to their ability to suppress IL-1 $\beta$-induced inflammation and cartilage degradation $[33,34]$. During disease progression, IL-1 $\beta$ stimulates the expression of the matrix metalloproteinase (MMPs) that mediate the degradation of cartilage matrix components and suppress proteoglycan synthesis [35]. Our study further revealed that UA obviously inhibited the levels of MMP3 and MMP13 enhanced by IL-1 $\beta$ treatment. IL-1 $\beta$ also promotes the expression of iNOS and COX-2, which induces the production of nitric oxide (NO) and prostaglandin E2 (PGE2), respectively. $\mathrm{NO}$ is a well-known inflammatory mediator that can induce MMP secretion and activation and decrease Collagen II and proteoglycan synthesis. In the present study, we found that IL-1 $\beta$-induced expression of iNOS and COX2 were also attenuated by UA. Sox-9 is a vital transcription factor that positively regulates Collagen II synthesis and is indispensable for chondrocyte differentiation [36]. Our study further pointed out that UA obviously restored the levels of SOX9 inhibited by IL$1 \beta$ treatment. To sum up, our data showed the therapeutic effect of UA by restoring the imbalance between anabolism and catabolism of the cartilage matrix.

A recent study also found that UA suppressed the excessive production of NO, PGE2, IL- 6 and TNF- $\alpha$ in collagenase-isolated human OA chondrocytes [19]. However, chondrocytes isolated from OA patients had showed various phenotypic changes due to destructive changes in the joint which does not seem to reflect the natural process of $\mathrm{OA}[37,38]$. In contrast, primary chondrocytes, especially cartilage explants in which chondrocytes remain in contact with the extracellular matrix, are more sensitive to molecular environment than OA chondrocytes, thus ex-vivo experiments based on primary chondrocytes and cartilage explants seem to be a more reliable OA-model [38]. In this study, we isolated rat chondrocytes as well as rat articular cartilage explant for experiments, which would provide further evidence that UA may inhibit IL-1 $\beta$-induced inflammatory response and preserve ECM of cartilage explant tissues.

Various intracellular signaling pathways are reported to participate in OA pathogenesis. The MAPK and NF$\mathrm{KB}$ pathways are master regulators of inflammation and catabolism in the process of OA. MAPK, mitogenactivated protein kinase, is a serine/threonine protein 


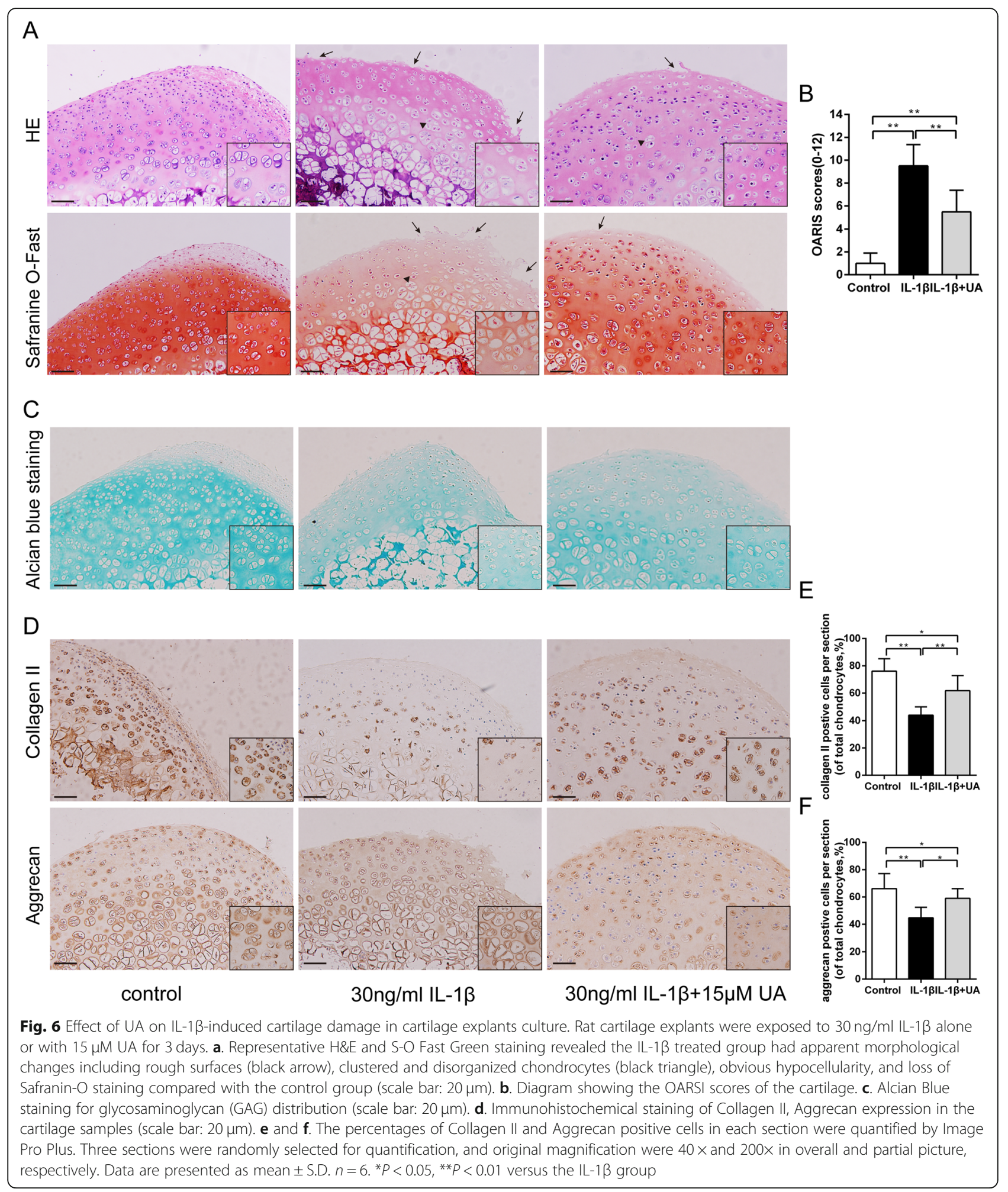

kinase in the cell that transmits extracellular stimulation signals into the cell, causing cellular responses such as cell proliferation, differentiation, transformation, and apoptosis. Under inflammatory stimulation, three major members of this pathway include extracellular regulated kinase (ERK), N-terminal kinase (JNK) and p38 are activated and the phosphorylation levels of them are upregulated, thus activating the downstream transduction pathway and then activating transcription factors, resulting in the release of pro-inflammatory factors and 


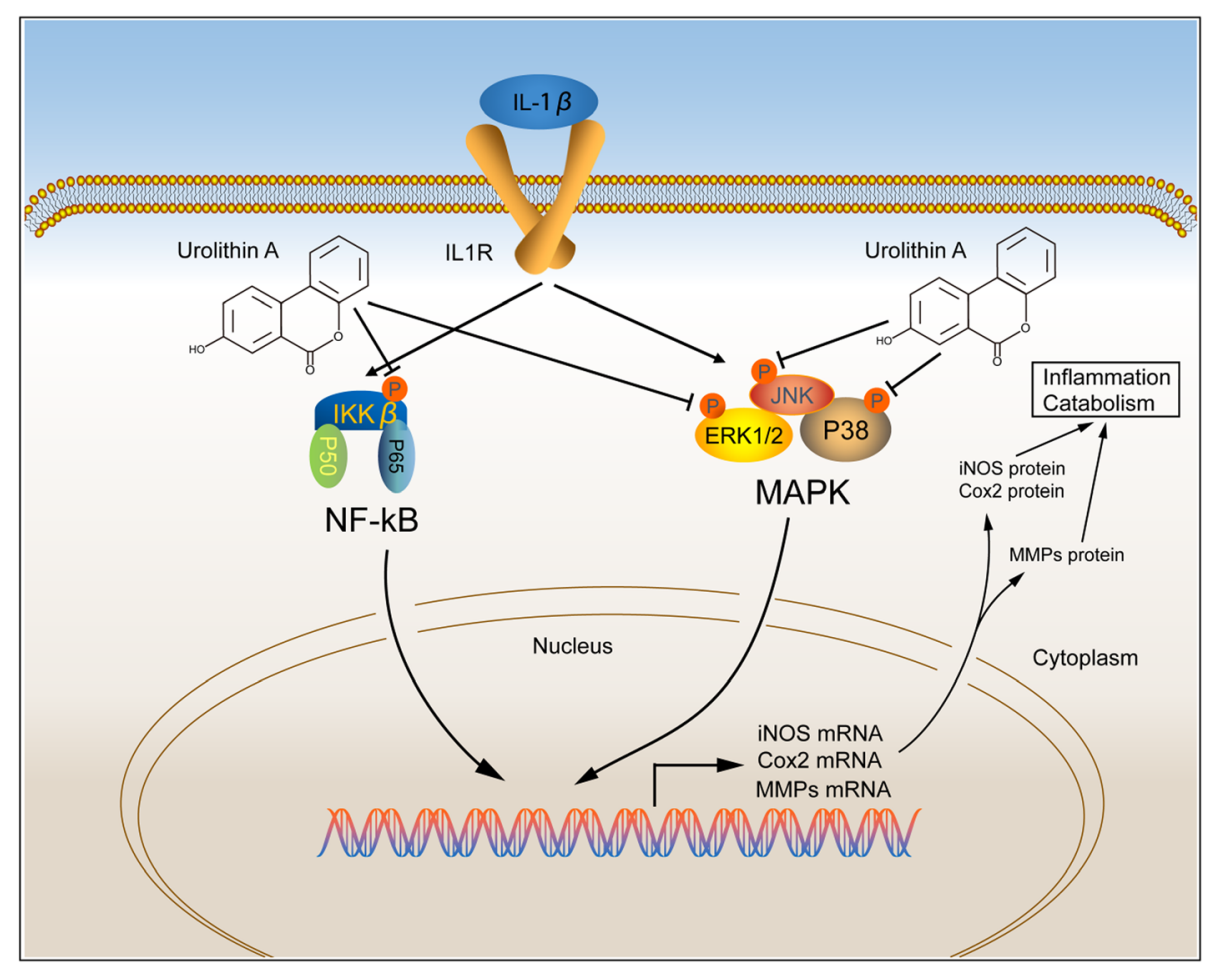

Fig. 7 A schematic of the proposed mechanism. During degeneration, MAPK and NF-KB signaling are activated by IL-1 $\beta$ stimulation. UA treatment can decrease iNOS, COX2, and MMPs expression and increase matrix gene expression through inhibiting activation of the MAPK and NF-KB pathways

inflammatory response. For OA, the active form of ERK, JNK and p38 was observed in synovial tissue and cartilage lesion. In addition, several lines of evidence understate that activation of MAPK induced by IL-1 $\beta$ trigger aggrecanases and MMPs-mediated articular cartilage degradation [36, 39]. Specially, Therapy targeting p38 inhibitors could attenuate cartilage degeneration and relief pain in animal models [40].

The NF- $\mathrm{kB}$ pathway includes a family of ubiquitously expressed transcription factors and regulates inflammatory responses $[17,41]$. Normally, the transcription factor exists in the cytoplasm and is render inactive by a constitutive interaction with the inhibitory protein ІкB. Once stimulated by IL- $1 \beta$, NF- $\mathrm{kB}$ p 65 translocates into the nucleus where it stimulates the expression of inflammatory mediators such as iNOS, COX-2, and MMPs, among these factors, iNOS catalyzes NO, which stimulates the secretion of MMPs and represses collagen II and proteoglycan synthesis to cause ECM degradation $[21,39]$. Previous studies demonstrated that UA could attenuate lipopolysaccharide-induced inflammation by inhibiting activation of the MAPK and NF-kB pathways and alleviate oxidized low-density lipoprotein-induced endothelial dysfunction by modulating MAPK signaling $[42,43]$. Our results demonstrated that IL- $1 \beta$ activated ERK, JNK, and p38; upregulated levels of phosphorylated p65; and increased nuclear translocation of p65. In articular cartilage, IL-1 $\beta$ binding to IL-1 receptor results in the recruitment of MyD88, followed by the activation of IRAKs and the E3 ubiquitin ligase TRAF6, and finally activate the MAPK and NF- $\mathrm{kB}$ pathway [44]. Furthermore, stimulation of IL-1 $\beta$ induces the accumulation of reactive oxygen species (ROS), known as second messengers during the activation of redox-sensitive transcription factors the MAPK and NF- $\mathrm{kB}$ pathway [45]. UA can exert its anti-inflammation and anti-oxidant activity effect through multiple ways. Although its specific target still needs to be explored, our data suggest that UA exerted its beneficial effects by inhibiting MAPK and NF- $\mathrm{kB}$ signaling (Fig. 7).

\section{Conclusions}

Our results provide evidence that UA can attenuate IL$1 \beta$-induced degradation of Collagen II and aggrecan and reduces the production of inflammatory mediators via the ERK, JNK, P38, and NF- $\mathrm{kB}$ pathways in rat chondrocytes. Collectively, these findings suggest that UA may be a promising therapeutic agent in the treatment of OA.

\section{Abbreviations}

ADAMTs: A disintegrin and metalloproteinase with thrombospondin type 1 motifs; Collagen II: Type II collagen; COX-2: Cyclooxygenase-2; 
ECM: Extracellular matrix; IL-1 $\beta$ : Interleukin-1 $\beta$; iNOS: Inducible nitric oxide synthase; MAPK: Mitogen-activated protein kinase; MMPs: Matrix metalloproteinases; NF-kB: Nuclear factor-kB; OA: Osteoarthritis; UA: Urolithin A

\section{Acknowledgments}

We are highly thankful to Dr. Fu-yong Wang for assistance with technical assistance and data analysis.

\section{Authors' contributions}

$\mathrm{ZR}, \mathrm{JH}, \mathrm{SD}$, and ZP conceived and devised the study. JH, RZ, and SD performed the experiments. $Z \mathrm{P}, \mathrm{XC}, \mathrm{ZL}$, and $\mathrm{XL}$ analyzed the data. JH, SD, and $\mathrm{PZ}$ wrote the paper. JH and QZ revised the manuscript. ZR obtained the funding and supervised the whole project. All authors have contributed to the final version and approved the publication of the final manuscript.

\section{Funding}

Financial supported from the Project of Qingpu District Science and Technology Commission (QKY2018-11) and the Project of Qingpu Branch of Zhongshan Hospital (QYZ2019-02 and QY2019-04).

\section{Availability of data and materials}

All data generated or analyzed during this study are included in this published article and are available from the corresponding author upon request.

\section{Ethics approval and consent to participate}

All animals received care in compliance with the Principles of Laboratory Animal Care and National standards.

\section{Consent for publication}

Not applicable

\section{Competing interests}

The authors declare that they have no conflict of interest.

\section{Author details}

'Department of Orthopaedic Surgery, Qingpu Branch of Zhongshan Hospital, Fudan University, 1158 Gong Yuan Dong Road, Qingpu District, Shanghai 201700, China. ${ }^{2}$ Department of Orthopaedic Surgery, Zhongshan Hospital, Fudan University, 180 Feng Lin Road, Xuhui District, Shanghai 200032, China. ${ }^{3}$ Department of Anesthesiology, Shanghai JiaoTong University Affiliated Shanghai Sixth People's Hospital, Shanghai 200233, China.

\section{Received: 30 October 2019 Accepted: 13 March 2020}

Published online: 24 March 2020

\section{References}

1. Chen D, Shen J, Zhao W, Wang T, Han L, Hamilton JL, Im HJ. Osteoarthritis: toward a comprehensive understanding of pathological mechanism. Bone Res. 2017:5:16044.

2. Roman-Blas JA, Jimenez SA. NF-kappaB as a potential therapeutic target in osteoarthritis and rheumatoid arthritis. Osteoarthr Cartil. 2006;14:839-48.

3. Dinarello CA. Immunological and inflammatory functions of the interleukin1 family. Annu Rev Immunol. 2009;27:519-50.

4. Bonnet CS, Walsh DA. Osteoarthritis, angiogenesis and inflammation. Rheumatology (Oxford). 2005;44:7-16.

5. van der Kraan PM, van den Berg WB. Chondrocyte hypertrophy and osteoarthritis: role in initiation and progression of cartilage degeneration? Osteoarthr Cartil. 2012;20:223-32.

6. Rahmati M, Mobasheri A, Mozafari M. Inflammatory mediators in osteoarthritis: a critical review of the state-of-the-art, current prospects, and future challenges. Bone. 2016;85:81-90.

7. Kobayashi M, Squires GR, Mousa A, Tanzer M, Zukor DJ, Antoniou J, Feige U, Poole AR. Role of interleukin-1 and tumor necrosis factor alpha in matrix degradation of human osteoarthritic cartilage. Arthritis Rheum. 2005;52:128-35.

8. Kihara S, Hayashi S, Hashimoto S, Kanzaki N, Takayama K, Matsumoto T, Chinzei N, Iwasa K, Haneda M, Takeuchi K, et al. Cyclin-dependent kinase Inhibitor-1-deficient mice are susceptible to osteoarthritis associated with enhanced inflammation. J Bone Miner Res. 2017;32:991-1001.
9. Kang I, Buckner T, Shay NF: Improvements in Metabolic Health with Consumption of Ellagic Acid and Subsequent Conversion into Urolithins: Evidence and Mechanisms 2016, 7:961-972. PMID:27633111.

10. Toney AM, Fan R, Xian Y, Chaidez V, Ramer-Tait AE, Chung S. Urolithin a, a gut metabolite, Improves Insulin Sensitivity Through Augmentation of Mitochondrial Function and Biogenesis. 2019;27:612-20. PMID:30768775.

11. Essa MM, Subash S, Akbar M, Al-Adawi S, Guillemin GJ. Long-term dietary supplementation of pomegranates, figs and dates alleviate neuroinflammation in a transgenic mouse model of Alzheimer's disease. PLoS One. 2015;10:e0120964.

12. Guada M, Ganugula R, Vadhanam M, Ravi Kumar MNV. Urolithin a mitigates Cisplatin-induced nephrotoxicity by inhibiting renal inflammation and apoptosis in an experimental rat model. J Pharmacol Exp Ther. 2017;363:58-65.

13. Saha P, Yeoh BS, Singh R, Chandrasekar B, Vemula PK, Haribabu B, VijayKumar M, Jala VR. Gut microbiota conversion of dietary Ellagic acid into bioactive Phytoceutical Urolithin a inhibits Heme peroxidases. PLoS One. 2016;11:e0156811.

14. Les F, Arbones-Mainar JM, Valero MS, Lopez V. Pomegranate polyphenols and urolithin a inhibit alpha-glucosidase, dipeptidyl peptidase-4, lipase, triglyceride accumulation and adipogenesis related genes in 3T3-L1 adipocyte-like cells. J Ethnopharmacol. 2018:220:67-74.

15. Norden $\mathrm{E}$, Heiss EH. Urolithin a gains in antiproliferative capacity by reducing the glycolytic potential via the p53/TIGAR axis in colon cancer cells. Carcinogenesis. 2019;40:93-101.

16. Liu H, Kang H, Song C, Lei Z, Li L, Guo J, Xu Y, Guan H, Fang Z, Li F. Urolithin a inhibits the catabolic effect of TNFalpha on nucleus Pulposus cell and alleviates intervertebral disc degeneration in vivo. Front Pharmacol. 2018;9:1043.

17. Rodriguez J, Caille O, Ferreira D, Francaux M. Pomegranate extract prevents skeletal muscle of mice against wasting induced by acute TNF-alpha injection. Mol Nutr Food Res. 2017;61. PMID:27804206.

18. Gonzalez-Sarrias A, Larrosa M, Tomas-Barberan FA, Dolara P, Espin JC. NFkappaB-dependent anti-inflammatory activity of urolithins, gut microbiota ellagic acid-derived metabolites, in human colonic fibroblasts. Br J Nutr. 2010;104:503-12.

19. Fu X, Gong LF, Wu YF, Lin Z, Jiang BJ, Wu L, Yu KH. Urolithin A targets the PI3K/Akt/NF-kappaB pathways and prevents IL-1 beta-induced inflammatory response in human osteoarthritis: in vitro and in vivo studies. 2019;10:6135-46. PMID:31497826.

20. Nishida T, Kubota S, Aoyama E, Yamanaka N, Lyons KM, Takigawa M. Lowintensity pulsed ultrasound (LIPUS) treatment of cultured chondrocytes stimulates production of CCN family protein 2 (CCN2), a protein involved in the regeneration of articular cartilage: mechanism underlying this stimulation. Osteoarthr Cartil. 2017;25:759-69.

21. Shimizu M, Matsumoto T, Kikuta S, Ohtaki M, Kano K, Taniguchi H, Saito S, Nagaoka M, Tokuhashi Y. Transplantation of dedifferentiated fat cell-derived micromass pellets contributed to cartilage repair in the rat osteochondral defect model. J Orthop Sci. 2018;23:688-96.

22. Yamashita A, Morioka M, Yahara Y, Okada M, Kobayashi T, Kuriyama S, Matsuda S, Tsumaki N. Generation of scaffoldless hyaline cartilaginous tissue from human iPSCs. Stem Cell Reports. 2015;4:404-18.

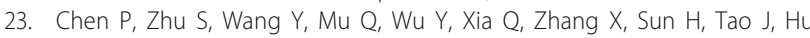
$\mathrm{H}$, et al. The amelioration of cartilage degeneration by ADAMTS-5 inhibitor delivered in a hyaluronic acid hydrogel. Biomaterials. 2014;35: 2827-36.

24. Pritzker KP, Gay S, Jimenez SA, Ostergaard K, Pelletier JP, Revell PA, Salter D, van den Berg WB. Osteoarthritis cartilage histopathology: grading and staging. Osteoarthr Cartil. 2006;14:13-29.

25. Glasson SS, Chambers MG, Van Den Berg WB, Little CB. The OARSI histopathology initiative - recommendations for histological assessments of osteoarthritis in the mouse. Osteoarthr Cartil. 2010;18(Suppl 3):S17-23.

26. Pang T, Wang J, Benicky J, Sánchez-Lemus E, Saavedra JM. Telmisartan directly ameliorates the neuronal inflammatory response to $\mathrm{IL}-1 \beta$ partly through the JNK/c-Jun and NADPH oxidase pathways. J Neuroinflammation,9,1(2012-05-29). 2012;9:102.

27. Kapoor M, Martel-Pelletier J, Lajeunesse D, Pelletier JP, Fahmi H. Role of proinflammatory cytokines in the pathophysiology of osteoarthritis. Nat Rev Rheumatol. 2011;7:33-42.

28. Ulici V, Kelley KL, Azcarate-Peril MA, Cleveland RJ, Sartor RB, Schwartz TA, Loeser RF. Osteoarthritis induced by destabilization of the medial meniscus is reduced in germ-free mice. Osteoarthr Cartil. 2018;26:1098-109. 
29. Sharon G, Cruz NJ, Kang DW, Gandal MJ, Wang B, Kim YM, Zink EM, Casey CP, Taylor BC, Lane Cl, et al. Human Gut Microbiota from Autism Spectrum Disorder Promote Behavioral Symptoms in Mice. Cell. 2019;177:1600-1618. e1617.

30. Donohoe DR, Garge N, Zhang X, Sun W, O'Connell TM, Bunger MK, Bultman $\mathrm{SJ}$. The microbiome and butyrate regulate energy metabolism and autophagy in the mammalian colon. Cell Metab. 2011;13:517-26.

31. Fukuda S, Toh H, Hase K, Oshima K, Nakanishi Y, Yoshimura K, Tobe T, Clarke $J M$, Topping DL, Suzuki T, et al. Bifidobacteria can protect from enteropathogenic infection through production of acetate. Nature. 2011; 469:543-7.

32. Que Q, Guo X, Zhan L, Chen S, Zhang Z, Ni X, Ye B, Wan S. The GLP-1 agonist, liraglutide, ameliorates inflammation through the activation of the PKA/CREB pathway in a rat model of knee osteoarthritis. J Inflamm (Lond). 2019:16:13.

33. Pirozzi C, Francisco V, Guida FD, Gomez R, Lago F, Pino J, Meli R, Gualillo O. Butyrate modulates inflammation in chondrocytes via GPR43 receptor. Cell Physiol Biochem. 2018;51:228-43.

34. Xu J, Yuan C, Wang G, Luo J, Ma H. Urolithins attenuate LPS-induced Neuroinflammation in BV2Microglia via MAPK, Akt, and NF-kappaB signaling pathways. J Agric Food Chem. 2018;66:571-80.

35. Goldring MB, Goldring SR. Osteoarthritis. J Cell Physiol. 2007;213:626-34.

36. Chen WP, Jin GJ, Xiong Y, Hu PF, Bao JP, Wu LD. Rosmarinic acid downregulates NO and PGE2 expression via MAPK pathway in rat chondrocytes. 2018;22:346-53. PMID:28945000.

37. Ji Q, Zheng Y, Zhang G, Hu Y, Fan X, Hou Y, Wen L, Li L, Xu Y, Wang Y, Tang F. Single-cell RNA-seq analysis reveals the progression of human osteoarthritis. Ann Rheum Dis. 2019;78:100-10.

38. Cope PJ, Ourradi K, Li Y, Sharif M. Models of osteoarthritis: the good, the bad and the promising. Osteoarthr Cartil. 2019;27:230-9.

39. Marcu KB, Otero M, Olivotto E, Borzi RM, Goldring MB. NF-kappaB signaling: multiple angles to target OA. Curr Drug Targets. 2010;11:599-613.

40. Brown KK, Heitmeyer SA, Hookfin EB, Hsieh L, Buchalova M, Taiwo YO, Janusz MJ. P38 MAP kinase inhibitors as potential therapeutics for the treatment of joint degeneration and pain associated with osteoarthritis. J Inflamm (Lond). 2008:5:22.

41. Baldwin AS Jr. Series introduction: the transcription factor NF-kappaB and human disease. J Clin Invest. 2001;107:3-6.

42. Piwowarski JP, Kiss AK, Granica S, Moeslinger T. Urolithins, gut microbiotaderived metabolites of ellagitannins, inhibit LPS-induced inflammation in RAW 264.7 murine macrophages. Mol Nutr Food Res. 2015;59:2168-77.

43. Komatsu W, Kishi H, Yagasaki K, Ohhira S. Urolithin a attenuates proinflammatory mediator production by suppressing PI3-K/Akt/NF-kappaB and JNK/AP-1 signaling pathways in lipopolysaccharide-stimulated RAW264 macrophages: possible involvement of NADPH oxidase-derived reactive oxygen species. Eur J Pharmacol. 2018;833:411-24.

44. Luo H, Liu Y, Li Q, Liao L, Sun R, Liu X, Jiang M, Hu J. A20 regulates IL-1induced tolerant production of CXC chemokines in human mesangial cells via inhibition of MAPK signaling. Sci Rep. 2015;5:18007.

45. Wang C, Yang Y, Zhang Y, Liu J, Yao Z, Zhang C. Protective effects of metformin against osteoarthritis through upregulation of SIRT3-mediated PINK1/Parkin-dependent mitophagy in primary chondrocytes. Biosci Trends. 2019;12:605-12.

\section{Publisher's Note}

Springer Nature remains neutral with regard to jurisdictional claims in published maps and institutional affiliations.

Ready to submit your research? Choose BMC and benefit from:
- fast, convenient online submission
- thorough peer review by experienced researchers in your field
- rapid publication on acceptance
- support for research data, including large and complex data types
- gold Open Access which fosters wider collaboration and increased citations
- maximum visibility for your research: over 100M website views per year
At BMC, research is always in progress.
Learn more biomedcentral.com/submissions

